This paper is published in the open archive of Mid Sweden University

DIVA http://miun.diva-portal.org

with permission of the publisher

Citation for the peer-reviewed published paper:

Gärdlund L, Forsström J, Andreasson B, Wågberg L. Influence of polyelectrolyte complexes on strength properties of papers made from unbleached kraft pulps. Nordic Pulp \& Paper Research Journal. $2005 ; 20(1): 36-42$.

URL to article at publishers site:

http://dx.doi.org/10.3183/NPPRJ-2005-20-01-p036-042 


\title{
Influence of polyelectrolyte complexes on the strength properties of papers from unbleached kraft pulps with different yields
}

\author{
Linda Gärdlund and Jennie Forsström, Fibre Science and Communication Network, Mid Sweden University, Bo Andreasson, SCA Graphic Research, \\ Sweden, Lars Wågberg, Department of Fibre and Polymer Technology, Royal Institute of Technology, KTH, Sweden
}

KEYWORDS: Adsorption, Polyelectrolyte complexes, Compression strength, Polyelectrolytes, Strength properties, Unbleached pulp, Pulp yield

SUMMARY: The properties of the materials combined in corrugated board are set to give the board its strength, flexibility and protection towards impact and pressure. The raw material is of course the single fibre but additives that enhance e.g. the wet and dry paper strength are also added. As the strength enhancing additives used today are most efficient between $\mathrm{pH} 4$ and $\mathrm{pH} 7$ there is a need for new types of additives that can be used under alkaline conditions. In the present report polyallylamine hydrochloride (PAH) and polyelectrolyte complexes (PEC) of PAH and polyacrylic acid (PAA) were investigated as strength enhancing additives. The components can be used under alkaline conditions and results showed that PAH alone or incorporated into a PEC gave different effects regarding strength properties of sheets from kraft pulps of different yields. The results showed that by treating the fibres with PEC's of PAH and PAA it was possible to considerably increase the tensile properties, the Z-strength and the compression strength of papers made from the treated fibres. The results showed an increase of as much as $54 \%$ to $180 \%$ in dry Z-strength and 14 $\%$ to $53 \%$ in compression strength, when using the PEC as an additive, indicating an increase in inter-fibre joint strength. It was also found that a heat treatment of the non-treated papers and of the papers from the PAH treated fibres gave a large improvement in tensile properties as well as Z-strength properties for the different pulps. For the PEC treated fibres it was not necessary to heat-treat the paper to achieve a higher dry strength. The molecular mechanism behind the large improvements is not identified in the present work but the problem is currently being investigated in the lab of the authors.

ADDRESSES OF THE AUTHORS: Linda Gärdlund (linda.gardlund@mh.se): Mid Sweden University, Department of Natural and Environmental Science, Holmgatan 10, SE85170 Sundsvall, Sweden. Jennie Forsström (jenfor@kth.se) and Lars Wågberg (wagberg@polymer.kth.se), Royal Institute or Technology, KTH, Department of Fibre and Polymer Technology, Div. Fibre Technology, SE-100 44 Stockholm, Sweden. Bo Andreasson (bo.andreasson@sca.com): SCA Graphic Research, SE-851 70 Sundsvall, Sweden.

Corresponding author: Linda Gärdlund

Liner and fluting are the two components of corrugated board and the properties of these materials are combined to give the board its strength, flexibility and protection towards impact and pressure. Different dry strength additives such as cationic starch and acrylamide polymers (Marton 1996) and wet strength additives such as polyamideamine epichlorohydrin resins (PAE) are therefore commonly used in the production of linerboard for strength enhancing purposes. One strategy of improving the strength of the paper and hence of the board is simply to add more of a desired chemical additive. However, the adsorption of an additive is limited by the charge of the fibres and the additive together with the ionic strength of the solution (Wågberg 2000). This means that at a certain level of addition, the surfaces are saturated and further additions of strength additives ends up in the white water causing a large variety of difficulties. A size press addition is naturally an alternative to wet end additions but this contributes to a significant capital cost and to an extra drying of the sheet.

It has long been debated weather it is the fibre-fibre joint or the single fibre strength that determines the strength utilised in the formed network. Davison (1972) found the limiting factor to be the joint strength between the fibres. Davison (1972) also showed that the fibre strength is at least twice as large as the strength of the sheet composed of these fibres. It has also earlier been shown (Häggkvist 1998) that different additives interact with the fibre and the fibre network differently. Small enough additives enter the porous structure of the fibre whereas larger additives only adsorb to the external fibre surface. When adding wet and dry strength additives it is therefore important to know whether the single fibre and/or the fibre-fibre joint should be strengthened. There is also a need for new types of additives, which can be used at alkaline conditions, since for example the cationic acrylamide resins have their main effect between pH 4 and pH 7 (Reynolds 1980).

For packaging material the dry strength in compression is of primary importance since corrugated containers most commonly fail in compression. The compression strength is initially determined by the joint strength but at a certain limit, the internal fibre wall strength starts to limit the compression strength between the fibres. To improve the compression strength the additives used must hence be able to diffuse into the fibre wall in order to increase its cohesive properties. In this respect the porous structure of the fibre wall is of significant importance since it limits the ability of the molecules of the additive to diffuse into the fibre wall. The porosity of the fibre wall also determines how much of the additive that adsorbs to the pulp (Stone et al 1968) and furthermore the ability of the fibres to conform to each other during consolidation of the paper. Wet strength in contrary to compression strength is mostly dependent on the joint strength as the limiting factor in wet strength is the water induced swelling of the fibre network. However, as shown earlier (Taylor 1968) the maximum in wet strength, possible to be achieved with a wet strength additive, is dependent on the ability of the additive to penetrate the fibre wall and prevent the moisture-induced separation of the concentric microfibrillar lamellae in the fibre wall. If only the external fibre surface is treated the wet paper will fail due to a peeling off of the external 
layers of the fibre wall (Taylor 1968). In this respect it is hence important to use additives that might penetrate the fibre wall and it is therefore vital to establish a relationship between the size of the polymer molecules of the additive and the size of the pores within the fibre wall.

Protection of the joints/bonds between the fibres is obtained by using additives that can cross-link with itself, hence forming an insoluble network around and through the fibre contacts (Dunlop-Jones 1996), or by creating permanent bonds between the fibres through the formation of covalent bonds between the additive and cellulose. The covalent bonds suppress the swelling of the fibres when the paper comes in contact with water and this can be achieved by adding polyelectrolytes, such as polyamines (Espy 1995; Taylor 1968). Cationic starch has been used to increase the joint strength between the fibres (Howard et al 1989) and studies have also been published where it has been claimed that this compound also increases the number of hydrogen bonds in a paper sheet (Moeller 1966). In (Rathi et al 2000) the polyallylamine hydrochloride was used as a dry strength additive. This additive can be used at $\mathrm{pH}$ levels higher than 7 and the polyelectrolyte was found to be a dry strength additive with high potential.

A polyelectrolyte multilayer technique was utilized to alter the surface properties of wood fibres for strength enhancing purposes (Wågberg et al 2002). A polyallylamine hydrochloride (PAH) was in this study combined with polyacrylic acid (PAA) to form polyelectrolyte multilayers on the fibres. These chemicals can furthermore form an amide linkage when exposed to heat. Another approach to the multilayer build-up is the pre-forming of polyelectrolyte complexes before addition to the pulp (e.g. Tsuchida et al 1982; Dautzenberg 1997). The complexes can, by its structural features, increase the molecular contact area in the fibre-fibre joint and in this way strengthen the joint. The use of polyelectrolyte complexes as strength enhancing additives was presented in (Gärdlund et al 2003) where the influence of complexes, with different total charges, on strength properties of papers made from fully bleached kraft pulps was investigated.

In the present study the aim was to investigate how the complexes between PAH and PAA behave as a strength enhancing additive, compared to the PAH alone, when added to well defined, unbleached, fines free, never dried kraft pulps. The pulps have different yields, charge properties and pore size distributions. The influence of heat treatment was also investigated.

\section{Experimental}

\section{Materials}

Chemicals. The cationic polyelectrolyte used in this study was a Polyallylamine hydrochloride, from Aldrich, with a molecular weight of $15000 \mathrm{~g} / \mathrm{mol}$ and $70000 \mathrm{~g} / \mathrm{mol}$, according to data from the supplier.

The anionic polyelectrolyte was Polyacrylic acid, from Aldrich, with a molecular weight of $8000 \mathrm{~g} / \mathrm{mol}$. Sodium chloride of analytical grade was used as a low molecular weight electrolyte. All chemicals were used without further purification and dissolved in and diluted with Milli Qwater prior to use. The Milli Q-water was obtained by purifying deionized water using a Milli $Q$ reagent-grade water system (Millipore, Eschborn, Germany).

Pulp preparation and pre-treatment. Three never dried, unbleached kraft pulps (yield 47.8 \%, $56.6 \%$ and 60.0 $\%)$ were used in the experiments. Wood chips from spruce were laboratory cooked according to a conventional kraft process in an 18-liter digester. The pulps with yield of $56.6 \%$ and $60.0 \%$ were prepared by treating the chips with $185-\mathrm{kg}$ effective alkali, EA, per tonnes dry weight, and the liquor: wood ratio was 3.2:1. The upper temperature $\left(160^{\circ} \mathrm{C}\right)$ of the cooking was reached after 100 minutes and the sulfidity was $35 \%$. The pulp with yield $47.8 \%$ was prepared by treating chips with 200-kg EA, per tonnes dry weight, and the liquor: wood ratio was $3.5: 1$. The cooking temperature was $161^{\circ} \mathrm{C}$ and the sulfidity was $35 \%$. After cooking the pulps were washed using deionized water and very mildly refined to liberate the fibres from the chips. Finally the pulps were de-watered to a dry content of about $25 \%$.

The fines were then removed to less than 0.5 weight $\%$ from the pulp by successive spraying through a spray disc filter equipped with a plastic wire with $75 \mu \mathrm{m}$ openings. The long fibre fraction was diluted to $3 \%$ fibre concentration and the $\mathrm{pH}$ value was adjusted to 2 with $\mathrm{HCl}$ and the suspension was kept under these conditions for 30 minutes. The pulp was then dewatered and diluted several times with deionized water until the $\mathrm{pH}$ reached 4.5. The carboxyl groups on the fibres were then converted to their $\mathrm{Na}^{+}$-form by treatment for 15 minutes with $0.01 \mathrm{M} \mathrm{NaHCO}_{3}$ and $\mathrm{NaOH}$ at $\mathrm{pH}$ 9. Finally the pulp was washed with deionized water to $\mathrm{pH}$ 7.5. These pulps were not allowed to dry before testing and were stored at $4^{\circ} \mathrm{C}$ at a solid content of $20 \%$.

\section{Methods}

Complex formation. The PAH with low molecular weight and the PAA were dissolved in Milli Q-water and the $\mathrm{pH}$ was adjusted to 7 . The polymer concentrations were 0.01 $\mathrm{M}$ with respect to charges and the concentration of $\mathrm{NaCl}$ was $0.01 \mathrm{M}$. The complexes were pre-formed at a mixing ratio of 0.8 , i.e the ratio between the added amounts of anionic to cationic charges $\left(\mathrm{q}^{-} / \mathrm{q}^{+}\right)$was 0.8 . The mixing procedure was carried out at $\mathrm{pH} 7$ using a titrator, 702 SM Titrino from Metrohm. After preparation the complex solution was filtered through a Millipore filter PTMK 000C5 with 300000 NMWL in order to remove the low molecular mass fraction of the excess cationic polyelectrolyte.

Charge and size determinations. The charge of the polyelectrolyte complexes was determined by the polyelectrolyte titration procedure outlined in (Wågberg et al 1989). To detect the point of charge neutralisation in the titration procedure a streaming current detector from Mütek $\mathrm{GmbH}$, Germany was used (Walker et al 1996).

The charge of the complexes was determined at $\mathrm{pH} 8$ since the adsorption and preparation of paper sheets was performed at $\mathrm{pH} 8$. To stabilize the $\mathrm{pH}$ of the solution during 
titration $10^{-4} \mathrm{M}$ of $\mathrm{NaHCO}_{3}$ (adjusted to $\mathrm{pH} 8$ ) was added.

The hydrodynamic average radius of the complexes was determined by dynamic light scattering. The dynamic light scattering gives an estimate of the diffusion of the complexes and this diffusion constant is then correlated to a hydrodynamic radius (Bordi et al 2003). The complexes were measured at $90^{\circ}$ scattering angle using a 90 -plus/BI-MAS equipment $(30 \mathrm{mV}$ diode laser, BI-9000AT correlator and a PMT detector) from Brookhaven Inc., Holtsville, NY, USA.

Charge determinations of the pulps. The charge of the fibres will influence the degree of swelling of the fibres and the ability of the fibres to adsorb charged polyelectrolytes or PEC's. It was therefore necessary to determine the surface charge of the fibres. This was done using the polyelectrolyte titration method outlined in (Wågberg et al) and a poly-diallyldimethylammoniumchloride (P-DADMAC) with a high molecular mass $(1.2 \cdot 106)$ was used in these measurements. The total charge of the fibres was determined by the conductometric titration method outlined in (Katz et al 1984).

Pore size distributions and degree of fibre swelling. The charges on the fibres will induce swelling of the fibres giving them a soft surface, which will be of importance for the obtained paper properties. The swelling behaviour was determined using WRV (Water Retention Value)measurements (Lindström 1986; Häggqvist et al 1998). Prior to these measurements the pulp was diluted in deionized water $(\mathrm{pH} \mathrm{8.0)}$ and then centrifuged at $3000 \mathrm{~g}$ for 15 minutes at $25^{\circ} \mathrm{C}$ (Häggqvist et al 1998), giving $\mathrm{A}$ WRV with a standard deviation (SD) of $\pm 4 \mathrm{ml} / \mathrm{g} * 100$. The fibres used for pore size distribution determinations were prepared by soaking the pulp for 15 minutes in $\mathrm{D} 2 \mathrm{O}$. The samples were then centrifuged according to the same procedure as used for the WRV preparation. The centrifuged pulp was then placed in 10-mm NMR tubes, which were sealed to prevent evaporation.

The ${ }^{2} \mathrm{H}$ NMR relaxation measurements were performed on a Bruker Avance DPX spectrometer (5.9 T magnet, ${ }^{2} \mathrm{H}$ resonance frequency $38.2 \mathrm{MHz}$ ). The spin lattice relaxation profiles were determined using the inversion recovery pulse sequence (Vold et al 1968). All experiments were performed at $22 \pm 0.5^{\circ} \mathrm{C}$. The ${ }^{2} \mathrm{H}$-pulse length was typically $25 \mu \mathrm{s}$. Each relaxation profile was recorded using about 30 delay times, $\tau$. All experiments were repeated and the SD was $\pm 0.3 \mathrm{~nm}$. The parameter $\beta$ was determined experimentally for two of the pulps (yield 60,8 \% and 48,1\%) using an earlier described method (Häggqvist et al 1998; Andreasson et al 2003). For the rest of the pulps the parameter b was set to be the same since no changes were made in the specific temperature and the magnetic field strength. The distribution of relaxation times and pore sizes were then calculated from the measured relaxation profiles using Contin (Provencher 1982).

Pulp preparation / Adsorption of polyelectrolytes and PEC's to the different pulps. The adsorption of cationic polyelectrolyte of low molecular weight and the PEC's was performed in deionized water with $0.01 \mathrm{M} \mathrm{NaCl}$ at $\mathrm{pH}$ 8. After 15 minutes of adsorption under continuous stirring the pulp was filtered on a nylon mesh wire and re- diluted in $0.01 \mathrm{M} \mathrm{NaCl}$ and $\mathrm{pH}$ 8. The amount of added compound was $3 \%(\mathrm{w} / \mathrm{w})$, to ensure a saturation of the fibres with the polyelectrolyte and the PEC's respectively.

The amount of adsorbed chemicals was determined by elemental nitrogen analysis using an Antek 7000, Antek Instruments Inc., Houston, USA. With this method the sample is pyrolized in air with a limited oxygen concentration and the NO formed reacts with ozone into excitated $\mathrm{NO}_{2}$ that, upon relaxation, will emit a lightquanta that can be used to determine the nitrogen content of the sample (Aksberg et al 1989). The polyelectrolyte titration method was also used to determine the adsorption of the PAH with two molecular weights to the pulps with different yields, here with respect to the difference in pore size distribution of the different pulps and its effect on adsorption. The adsorption was performed at $\mathrm{pH} 8$ in $0.01 \mathrm{M} \mathrm{NaCl}$ and the titration procedure was performed at $\mathrm{pH} 2$ in order to fully charge the polyelectrolytes and avoid the use of a buffer solution interfering with the measurements.

Sheet preparation and testing. Standard ISO sheets were prepared with a grammage of $120 \mathrm{~g} / \mathrm{m}$ ? in tap water. To be able to determine the effect of the covalent bonds between the fibres, treated and non-treated, half of the sheets were cured in $160^{\circ} \mathrm{C}$ for 15 minutes. The papers were then tested with respect to dry tensile strength properties (SCAN-P: 67) with a SD of $\pm 3 \mathrm{Nm} / \mathrm{g}$, Z-strength (delamination) properties (SCAN-P80:98) with a SD of \pm 20 $\mathrm{kN} / \mathrm{m}^{2}$, along with compression strength (SCT) ( SCANC 36:84) were the SD was $\pm 2 \mathrm{Nm} / \mathrm{g}$

The wet tensile strength properties was determined according to standard method (SCAN-P20:95) although the treated sheets were soaked in water for 15 seconds, which was enough to achieve a water content of the sheets of at least $60 \%$, the SD was $\pm 1 \mathrm{Nm} / \mathrm{g}$.

\section{Results and Discussion}

\section{Complex characterisation}

The polyelectrolyte titration procedure yielded a charge density of $1.8 \mathrm{meq} / \mathrm{g}$ of the complexes at $\mathrm{pH} 8$ and the obtained hydrodynamic mean radius of the PEC was 80$95 \mathrm{~nm}$. The charge of the PAH was $9 \mathrm{meq} / \mathrm{g}$.

\section{Pulp characterisation}

The results from the total and surface charge measurements along with WRV on untreated pulp can be seen in Table 1 .

As a comparison the WRV for a complex treated, never heated pulp with yield $56.6 \%$ is also shown.

As mentioned in the introduction the charge of the fibres has a significant influence on the swelling of the fibres. As can be seen in table 1 an increase in pulp yield gave an increase in total charge and swelling, this is in agreement with earlier published data for similar pulps (Andreasson et al 2003; Swerin and Wågberg 1994). The surface charge showed a maximum at a pulp yield of 56.6 $\%$ as can also be seen in Table 1. This maximum in surface charge has been found earlier (Swerin and Wågberg 1994) and was ascribed to be due to a balance between the contraction of the fibre structure, due to 


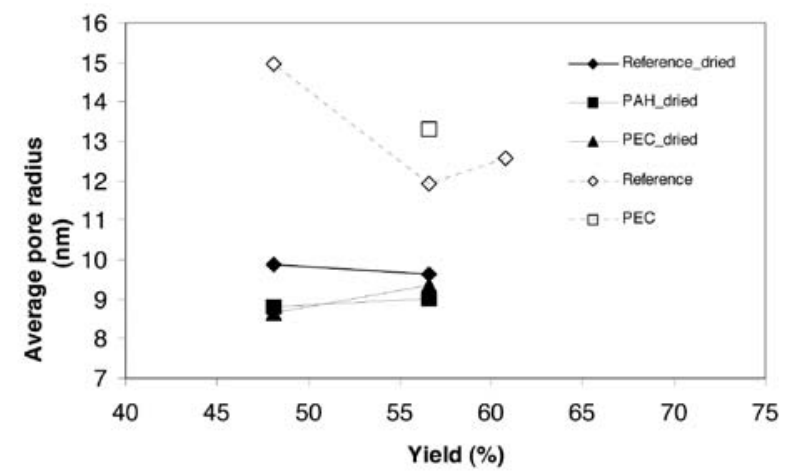

Fig 1. The average pore radius of the pulps with different yields and after different treatments

removal of lignin substances, and the repulsion between the charges within the fibre wall.

The average pore radius of the fibre wall was also determined for the differently treated fibres since a change in pore size of the treated pulps compared with the reference can give an indication whether the additives might have diffused into the fibre wall or not.

In Fig 1 the average pore radius for both dried and non-dried, treated and non-treated pulps with different yields is shown.

As can be seen in Fig 1 the average pore radius was $12-13 \mathrm{~nm}$ for the pulps with a yield between 55 and $61 \%$ and these results are in general agreement with the results shown in (Andreasson et al 2003; Maloney et al 1997). As the yield decreased further an increase in pore radius to about $15 \mathrm{~nm}$ was found.

This increase in pore radius is in general agreement with earlier published results (Andreasson et al 2003) although the increase was not as significant in the present investigation.

When the pulps were dried, the average pore radius decreased to about $10 \mathrm{~nm}$ and $9.5 \mathrm{~nm}$ for the yields 47.8 $\%$ and $56.6 \%$, respectively, due to irreversible closure of some pores within the fibre wall. When PAH and PEC treated fibres were dried the average pore radius were even more reduced. For the low yield pulp, average pore radius decreased from about $10 \mathrm{~nm}$ to about $8.7 \mathrm{~nm}$. This difference is definitely outside the limit of experimental error, which is only a few percent. However, for the high yield pulp, the average pore radius decreased from 9.5 $\mathrm{nm}$ to about $9.2 \mathrm{~nm}$. These values are within the limit of experimental error. Hence, it seems as both PAH and PEC were able to penetrate the fibre wall of the fibres of the low yield pulp to same extent. This indicates that the additives were able to change the wet/dry strength properties of the fibres as such. Possible improvements in wet and dry strength of the paper were hence also due to a strengthening of the fibre wall and not only by an increase of the joint strength between the fibres. It must be pointed out that no specific measurements reflecting fibre properties, such as zero span tensile testing, were conducted in the present investigation.

\section{Adsorption of PAH and PEC on the pulps of different yields}

In Table 2 the adsorption results as determined with the PET technique are shown. The adsorbed amount of low and high molecular weight PAH along with PEC from the nitrogen adsorption is shown in Table 3. The large difference between the adsorption values from PET measurements and from nitrogen analysis might be explained by the way these entities are evaluated. The PET measurements are evaluated from adsorption isotherms by extrapolating to zero polymer concentration, this in order to estimate a saturation adsorption on the fibre surfaces. However, since there is a positive slope of the isotherm a higher polymer addition leads to a higher adsorbed amount. For the nitrogen adsorption measurements a high addition of polymer was used $(30 \mathrm{mg} / \mathrm{g})$ and by extrapolating the adsorption isotherms from PET measurements to higher adsorbed amounts the nitrogen adsorption and the PET measurements yield similar results. The difference noted between Tables 2 and 3 is simply due to the fact that different parts of the adsorption isotherm are compared. In Table 2 it can be seen that for the pulps with yield $56.6 \%$ and $60.8 \%$ the adsorbed amount of PAH was almost the same regardless of yield and regardless of the molecular weigth of PAH although the high yield pulp adsorbed slightly more of the low molecular weight PAH than pulps with lower yield. For the low yield pulp though it can be seen that the adsorbed amount of low molecular weight PAH was approximately $35 \%$ higher than the adsorbed amount of high molecular weight PAH.

When determining the adsorbed amount of PAH in the formed sheets using nitrogen analysis the results were slightly different. The adsorption of low molecular mass PAH was the same regardless of pulp yield as seen in Table 3 and the amount of adsorbed charges was calculated to be $45 \mu$ eq./g irrespective of fibre charge. The exact explanation for this is not known but with support from the polyelectrolyte titration results a possi-

Table 1. Total- and surface charge of the fibres along with WRV for the pulps with different yield. * $=$ value not measured.

\begin{tabular}{llll}
\hline Yield (\%) & 47.1 & $\mathbf{5 6 . 6}$ & $\mathbf{6 0 . 8}$ \\
\hline Total charge $(\mu \mathrm{eg} / \mathrm{g})$ & 78 & 156 & 204 \\
Surface charge $(\mu \mathrm{eg} / \mathrm{g})$ & 2.7 & 5.6 & 5.0 \\
WRV $\left(\mathrm{ml} / \mathrm{g}^{*} 100\right)$ & 169 & 178 & 184 \\
WRV $\left(\mathrm{ml} / \mathrm{g}^{*} 100\right)$ when & & 187 & $*$ \\
treated with complex & $*$ & &
\end{tabular}

Table 2. Adsorbed amount PAH (Mw $15000 \mathrm{~g} / \mathrm{mol}$ and $\mathrm{Mw} 70000 \mathrm{~g} / \mathrm{mol}$ ) as determined with polyelectrolyte titration for the pulps with different yield.

\begin{tabular}{llll}
\hline Yield (\%) & 47.1 & 56.6 & 60.8 \\
\hline Adsorbed amount PAH & 2.7 & 2.7 & 3.0 \\
Mw $15000 \mathrm{~g} / \mathrm{mol}(\mathrm{mg} / \mathrm{g})$ & & & \\
PET & & 2.7 & 2.8 \\
Adsorbed amount PAH & 2.0 & & \\
Mw $70000 \mathrm{~g} / \mathrm{mol}(\mathrm{mg} / \mathrm{g})$ & & & \\
PET & &
\end{tabular}

Table 3. Adsorbed amount PEC and PAH (Mw $15000 \mathrm{~g} / \mathrm{mol}$ and Mw 70000 $\mathrm{g} / \mathrm{mol}$ ) as determined with nitrogen analysis for the pulps with different yield.

\begin{tabular}{llll}
\hline Yield (\%) & $\mathbf{4 7 , 1}$ & $\mathbf{5 6 , 6}$ & $\mathbf{6 0 , 8}$ \\
\hline $\begin{array}{l}\text { Adsorbed amount PEC (mg/g) } \\
\text { Nitrogen analysis }\end{array}$ & 12 & 10 & 6 \\
$\begin{array}{l}\text { Adorbed amount PAH (mg/g) } \\
\text { Nitrogen analysis }\end{array}$ & 5.2 & 5.5 & 4.7 \\
\hline
\end{tabular}


ble explanation is given below. As mentioned earlier the porous structure of the fibres will influence the adsorption of the additives combined with additive/pulp charge interactions. As the pulp yield is decreased the pore size of the fibres is increased and this leads to an opening-up of the structure that in turn will increase the availability of the fibre wall charges to the polyelectrolyte (in this study low $\mathrm{M}_{\mathrm{w}} \mathrm{PAH}$ ). The increase in pore size will result in a larger apparent charged surface in relation to the polyelectrolyte. This is also valid for the fraction of the PEC with a size smaller than the size of the high molecular weight polyelectrolytes normally used for standard surface charge determination. On the other hand a decrease in pulp yield is also followed by a decrease in the charge of the fibres. The increase in availability will hence be counteracted by the decrease in pulp charge and obviously these two effects will cancel each other out. This leads to the conclusion that despite a similarly adsorbed amount the PAH is differently distributed throughout the fibre wall. The PAH might reach deeper into the fibre wall for the fibres with a lower yield and in turn this might result in a different efficiency of the PAH to affect the pore size of these fibres as seen in Fig 1 .

The adsorption of PEC, as determined by nitrogen analysis, was highly dependent on pulp yield, as seen in Table 3. The high yield pulp adsorbed only half the amount of what was adsorbed by the low yield pulp. When recalculating the data shown in Table 3 it was found that the amounts of PEC charges adsorbed were $21.6 \mu \mathrm{eq} . / \mathrm{g}$ on the low yield pulp, $18.0 \mu \mathrm{eq} . / \mathrm{g}$ for the pulp with yield $56.6 \%$ and $10.8 \mu \mathrm{eq} . / \mathrm{g}$ for the high yield pulp, even though the surface charge of the low yield pulp was $\sim 50 \%$ less than the pulps with higher yield. From this it is obvious that the adsorbed amount of PEC charges is not only dependent on the fibre surface charges. In accordance with the discussion regarding the PAH adsorption, the adsorbed amount of PEC will also depend on the porosity of the fibre wall surface. A low yield pulp will have much more open porous surface structure than a high yield pulp, hence the adsorbed amount of PEC will increase when the pulp yield is decreased. Upon comparing the adsorptive behaviour of PAH and PEC it seems reasonable to suggest that the adsorption mechanisms of these two additives differ. The PEC and the PAH will hence be differently distributed throughout the fibre wall. In turn this will result in a different effect on the obtained paper properties of the two types of additives simply from the distribution of the additives over the fibre wall and fibre surface.

\section{Influence of PAH/PEC and heat treatment on paper properties}

In Figs 2-5, different strength properties of papers made from the pulps of different yields and with different treatments are shown. The treatments correspond to the addition of PAH and PEC and furthermore the influence of heat treatment is shown. In Figs 2-5 it can be seen that the addition of PAH and PEC resulted in large dry and wet strength improvements and that the strength could be even further increased by heat treatment. As mentioned earlier the increase in strength can be caused either by a strengthening of the fibre wall or by an increased joint strength between the fibres. The latter effect is naturally more important and also limiting for wet strength than for dry strength. It is of course difficult to draw any final conclusions from the present results, i.e. which of these two mechanisms that is the most important but the results give some interesting indications to what could be the probable explanations.

In Fig 2 the influence of the different treatments on the dry tensile strength properties as a function of pulp yield is shown. Papers made from the pulp with yield $56.6 \%$ showed the overall highest paper strength, irrespective of treatment with additives and heat. The occurrence of this maximum is most likely due to a combination of high swelling ability and conformability of the fibres together with a fibre wall that still contains enough lignin giving it strength (Andreasson et al 2003). The high yield pulp has a higher content of lignin making the fibre wall stiffer and less conformable. So even though the pulp had a high total charge the dry strength properties could not be fully utilised. The low yield reference pulp had a low charge hence its swelling ability was diminished and the strength properties of the formed paper decreased. It should also be noted that all the reference sheets showed the same behaviour upon exposure to heat, i.e. the tensile strength index increased.

In Fig 2 it can also be seen that the addition of PAH gave an increase in dry tensile strength and that the increase was highest for the low yield pulp closely followed by the pulp with highest yield. The pulp with yield 56.6\% was hardly affected at all by the addition of PAH and the reason for this is not clear. It can also be noted that upon heating the PAH treated sheets there was an increase in dry tensile strength. This increase is most probably due to the formation of amide linkages formed between the fibres and the additive. The addition of the PEC also resulted in an increase in dry tensile index and again the highest increase was found for the low yield pulp. The effect of heat treatment gave an additional $6 \%$ in dry tensile strength index increment, i.e. the complexes showed the same response towards heat treatment independent of the pulp yields.

It is obvious from the results shown in Fig 2 that the low yield pulp was more affected by the addition of PAH and PEC than the other pulps, with a significant higher tensile index when using PEC as an additive. Earlier it has been suggested that PAH penetrates into the fibrous structure of the pulp with low yield and it is therefore tempting to suggest that the effect of the PAH for these fibres is hence due to and increase of the bonding within the fibre wall. Naturally the PAH would also have an effect on the strength of the fibre-fibre joint but it might be suggested that the PEC has a much higher relative effect on the fibre-fibre joint strength since the PEC is not able to diffuse into the fibre wall to the same extent. This hypothesis is also supported by the fact that the PEC had a large effect on the development of dry tensile strength without heating but the sheets containing PAH needed heat treatment to get a high strength improvement. It must be emphasised though that there was a difference in adsorbed amount of the additives. 


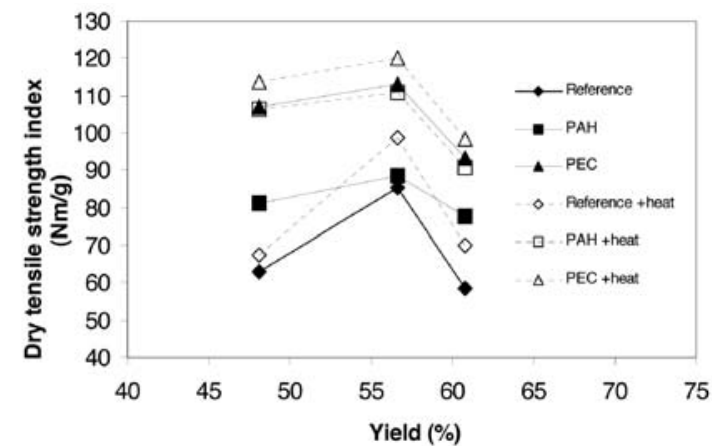

Fig 2. The dry tensile index as a function of pulp yield for papers made of pulps with different yield, with PAH and PEC as additives and with heat treatment along with no heat treatments.

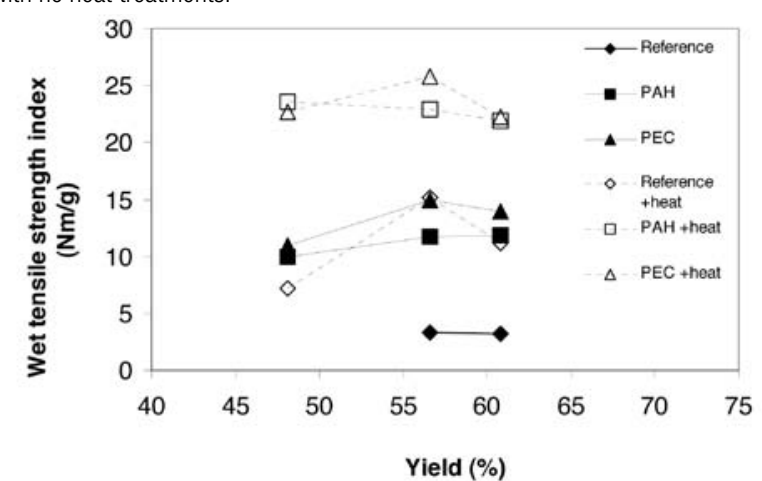

Fig 3. The wet tensile index as a function of pulp yield for papers made of pulps with different yield, with PAH and PEC as additives and with heat treatment along with no heat treatments.

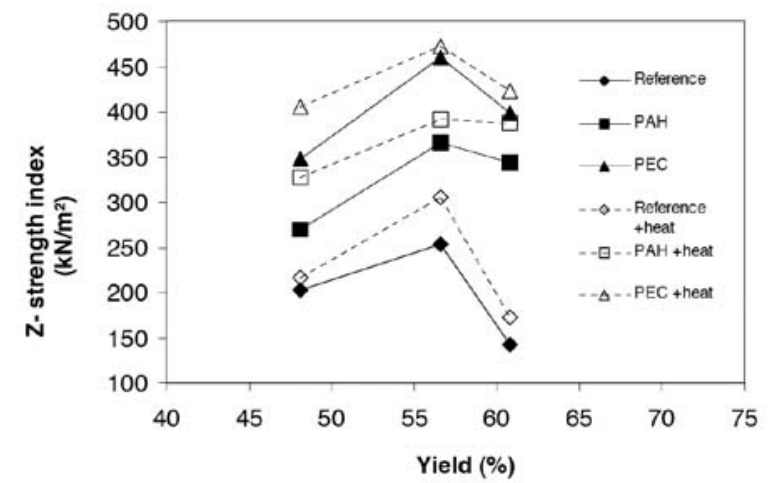

Fig 4. The Z-strength as a function of pulp yield for papers made of pulps with different yield, with PAH and PEC as additives and with heat treatment along with no heat treatments.

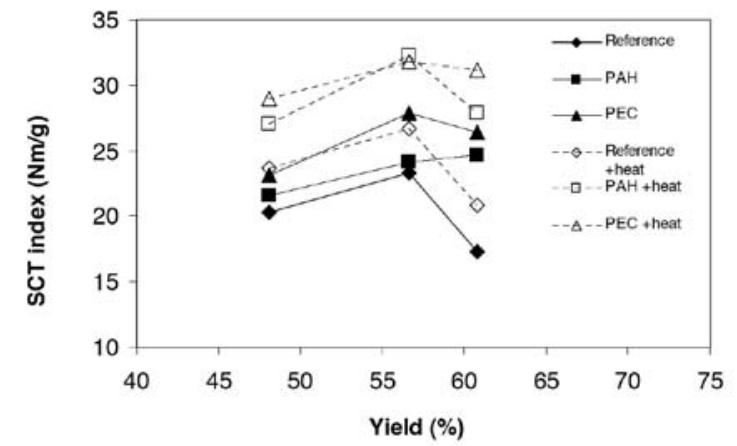

Fig 5. The compression strength as a function of pulp yield for papers made of pulps with different yield, with PAH and PEC as additives and with heat treatment along with no heat treatments.

Fig 3 shows the results from the measurements of the wet tensile index of the sheets corresponding to the same treatments as shown in Fig 2. Again papers made from pulp with yield $56.6 \%$ showed the overall highest paper strength, independent of treatment with additives and heat although the maximum is not as obvious as it was for the dry tensile strength. The treatments gave different results whereas the effect of heat treatment followed the same trend as noted for the dry tensile strength, i.e. heat treatment resulted in a paper with a higher wet strength. The most significant effect was obtained with the PEC and PAH upon exposing the papers to heat. This clearly displays the importance of covalent linkages, formed by the PAH itself or incorporated in PEC, for the development of wet strength. The PEC accompanied by heat treatment gave a maximum in tensile strength index of $27 \mathrm{Nm} / \mathrm{g}$. For pulps with yield $48.1 \%$ and $60.8 \%$ there was no difference in wet tensile index between the PAH and the PEC treatments. For pulp with yield 56.6\% the addition of PEC resulted in a somewhat higher index. The obtained wet strength results were however satisfying even without the heat treatment, which is important since the $160^{\circ} \mathrm{C}$ treatment is not directly applicable in practice.

The importance of the additives on the formation of strong joints between the fibres could also be detected with measurements of Z-strength properties. As shown in Fig 4 all the different treatments increased the Z-strength. The results followed the same trend as the tensile strength, with the maximum in Z-strength at $56.6 \%$ pulp yield for both non heat treated and heat treated sheets. It is however also clear from Fig 4 that the PAH treatments, heated or non-heated, was not as efficient as the PEC treatments and this strengthens the earlier hypothesis somewhat that the PAH is more efficient through an improvement of the intra-fibre bonding than through the formation of strong joints between the fibres.

Fig 5 shows the results from the measurements of the compression strength (SCT index) of the sheets corresponding to the same treatment as shown in the previous figures. The PEC treatment resulted in the overall highest compression strength, independent of pulp yields and post treatment of the papers. Contrary to the earlier presented strength parameters of the sheets, a PEC addition followed by heat treatment was sufficient enough to make the pulp with the highest yield almost as strong as the other pulps. This result is intriguing since it indicates that the additives in combination with a higher pulp yield can give products with superior compression properties.

There was also a significant difference in response to the different types of treatments for the fibres with different yields. The pulp with the lowest yield showed the highest response to the heat treatment whereas the fibres with the highest yield showed a higher response to the addition of the chemicals. This could be taken as an indication that the pulp with the lowest yield needed an increase in internal bonding within the fibre wall to improve the compression properties whereas the fibres with the highest yield were stiff enough from the remaining lignin in the fibre wall and could benefit most from an improved joint strength to enhance the compression properties.

It can also be suggested from the results that the stiffness, lost upon cooking, for the fibres from the low yield pulp could not be fully compensated using the additives followed by heat treatment since the SCT value for paper made from these fibres was lower than the 
value for the fully treated pulp with the highest yield. This also suggests that as the yield decreased, and the pores became larger, there was a shifting of the location of the additive in question to the interior of the fibre wall. In turn this leads to a shifting of the mechanism behind the action of the additives from a dependence of the contact area between the fibres creating an increased joint strength for the fibres with the highest yield towards a fibre wall stiffening and an increase in bond strength in the contact zone between the fibres due to a covalent linkage for the low yield pulps. This hypothesis needs further testing and a detailed action of polyelectrolyte complexes as strength additives on a molecular scale is under continuous investigation in our laboratories.

\section{Conclusions}

The present work has shown that the addition of PAH or PEC's of PAH and PAA can significantly increase the wet and dry strength properties of paper made from unbleached softwood pulps with different yields. It was suggested that PEC and PAH were differently distributed throughout the fibre wall and that this resulted in the different sheet properties obtained. In measurements of sheet tensile strength and Z-strength a maximum was seen for pulp with yield $56.6 \%$. The maximum was ascribed to be due to a combination of high swelling ability and conformability, together with a fibre wall that still contained enough lignin giving it strength. The addition of PEC significantly improved the tensile properties and the Z-strength properties of the papers without heat treatment whereas the PAH treated sheets needed a heat treatment to achieve an improvement similar to the PEC. In order to create large improvements in wet strength it was necessary to heat-treat also the PEC treated sheets. The compression strength data suggested a dependence on the development of covalent linkages within the fibre wall following the heat treatment of the papers especially for the low yield pulp. When increasing the yield of the pulps the dependence of improving the joint strength increased.

Adsorption measurements indicated that the low $\mathrm{M}_{\mathrm{w}}$ PAH penetrated deeper into the fibre wall of the fibres with the lowest yield. This resulted in a larger strength improvement following PAH addition and heat treatment for the pulps with the lowest yield.

The results also suggest that the effect of the PEC was dominated by an improvement in the strength of the fibre-fibre joint whereas the PAH effect was dominated by an improvement of the intra-fibre bond strength.

\section{Acknowledgements}

The authors would like to thank the Kempe Foundation and SCA for financial support and Dr Shannon Notley for linguistic revision of the manuscript.

\section{Literature}

Aksberg, R. and Wågberg, L. (1989): "Hydrolysis of cationic polyacrylamide"; J. Appl. Polym. Sci. 38(2), 297
Andreasson, B., Forsström, J. and Wågberg, L. (2003): "The porous structure of pulp fibres with different yields and its influence on paper strength"; Cellulose 10(2), 111. Bordi, F., Cametti, C., Gili, T., Gaudino, D. and Sennato, S. (2003): "Time evolution of the formation of different size cationic liposome-polyelectrolyte complexes"; Bioelectrochemistry 59(1-2), 99.

Dautzenberg, H. (1997): "Polyelectrolyte Complex Formation in Highly Aggregating Systems. 1. Effect of Salt: Polyelectrolyte Complex Formation in the Presence of NaCl"; Macromolecules 30(25), 7810

Davison, R. W. (1972): "The weak link in paper dry strength"; Tappi. 55(4), 567 Dunlop-Jones, N. (1996): In "Paper chemistry", 2nd edition, Edited. J. C Roberts, Blackie Academic \& Professional, Glasgow, p98

Espy, H. H. (1995): "The mechanism of wet-strength development in paper: a review"; Tappi J. 78(4), 90.

Gärdlund, L., Wågberg, L. and Gernandt, R. (2003): "Polyelectrolyte complexes for surface modification of wood fibres. -II. Influence of complexes on wet and dry strength of paper"; Colloids and Surfaces A: Physicochemical and Engineering Aspects, 218(1-3), 137.

Howard, R. C. and Jowsey, C. J. (1989): "The effect of cationic starch on the tensile strength of paper"; J.Pulp Pap. Sci. 15(6), 225.

Häggqvist, M., Li, T-Q. and Ödberg G, L. (1998): "Effects of drying and pressing on the pore structure in the cellulose fibre wall studied by $1 \mathrm{H}$ and $2 \mathrm{H}$ NMR relaxation"; Cellulose 5(1), 33.

Katz, S., Beatson, R. P. and Scallan, A. M. (1984): "The determination of strong and weak acidic groups in sulfite pulps"; Sv. Papperstidn. 87(6), 48

Lindström, T. (1986): In "Paper structure and properties", Edited J. A. Bristow and P. Kolseth, Marcel Dekker Inc, New York, p75.

Maloney, T., C., Li, T.-Q., Weise, U. and Paulapuro, H. (1997): "Intra- and interfibre pore closure in wet pressing"; Appita J. 50(4), 301.

Marton, J. (1996): In "Paper chemistry", 2nd edition, Edited. J. C. Roberts, Blackie Academic \& Professional, Glasgow, p83.

Moeller, H. W. (1966): "Cationic starch as a wet end strength additive"; Tappi , 49(5), 211.

Provencher, S. W. (1982): "A constrained regularisation method for inverting data represented by linear algebraic or integral equations", Comput. Phys. Commun. 27(3), 213

Rathi, M. S. and Biermann, C. J. (2000): Application of polyallylamine as a dry strength agent for paper"; Tappi J. 83(12), 62.

Reynold, W. F. (1980): "Acrylamide-based polymers for dry strength improvement of paper"; In Dry strength additives, ed. W. F. Reynolds, 125-148. TAPPI Press, Atlanta.

Stone, J. E. and Scallan, A. M. (1968): "A structural Model for the cell wall of water swollen wood pulp fibres based on their accessibility to macromolecules"; Cellulose Chem. Technol. 2(3), 343.

Swerin A. and Wågberg, L. (1994). "Size-exclusion chromatography for characterization of cationic polyelectrolytes used in papermaking"; Nordic Pulp Pap. Res. J. 9(1), 18.

Taylor, D. (1968): "Mechanism of wet tensile failure"; Tappi. 51(9), 410.

Tsuchida, E. and Abe, K. (1982): Interactions between macromolecules in solution and intermolecular complexes, Springer Verlag, Berlin.

Vold, R. L., Waugh, J. S., Klein, M. P. and Phelps, D. E. (1968): "Measurements of spin relaxation in complex systems"; J. Phys. Chem. 48(-), 3831.

Walker, C., Kirby, J. and Dentel, S. (1996): "The streaming current detector: a quantitative model"; J. Colloid. Interf. Sci. 182(1), 71.

Wågberg, L., Forsberg, S., Johansson, A. and Juntti, P. (2002): "Engineering of fibre surface properties by application of the polyelectrolyte multilayer concept. Part I: Modification of paper strength"; J. Pulp Pap. Sci. 28(7), 222.

Wågberg, L. (2000) "Polyelectrolyte adsorption onto cellulose fibres: a review"; Nord. Pulp Pap. Res. J. 15(5), 586.

Wågberg, L., Ödberg, L. and Glad- Nordmark, G. (1989): "Charge determinations of porous substrates by polyelectrolyte adsorption"; Nord. Pulp Pap. Res. J. $4(2), 71$

Manuscript received October 24, 2003 Accepted October, 2004 